\title{
Obesidad como Factor de Riesgo para Trastornos Metabólicos en Adolescentes Mexicanos, 2005
}

\author{
Obesity as risk factor for metabolic disorder in Mexican \\ adolescents, 2005
}

Nydia J. Marcos-Daccarett ${ }^{1}$, Georgina M. Núñez-Rocha ${ }^{1}$, Ana M. Salinas-

Martínez $^{1}$, Magaly Santos-Ayarzagoitia ${ }^{2}$ y Horacio Decanini-Arcaute ${ }^{1}$

1. Instituto Mexicano del Seguro Social. Monterrey Nuevo León, México.njmarcosd@hotmail.com, gmnunezr@cis.gob.mx, amsalinasm@intercable.net

2. Hospital Universitario. Universidad Autónoma de Nuevo León. Monterrey, México

Recibido 5 Octubre 2006/Enviado para Modificación 25 Marzo 2007/Aceptado 30 Abril 2007

\section{RESUMEN}

Objetivo Determinar la prevalencia y estimar el riesgo de obesidad para dislipidemias e hiperinsulinemia en adolescentes. Asimismo, evaluar la asociación lineal de medidas antropométricas y lípidos con insulina.

Material y Métodos Se realizó un estudio transversal comparativo entre adolescentes obesos (índice de masa corporal=IMC>percentil 95 para edad y sexo; $n=120$ ) y no obesos (IMC<percentil 85 para edad y sexo; $n=120$ ) de 10-19 años. Se aplicó una encuesta estructurada con datos sociodemográficos y antropométricos y se determinaron glucosa, insulina y perfil de lípidos.

Resultados La prevalencia de una o más dislipidemias fue de $56,6 \%$ en adolescentes c/obesidad, en comparación con $20,8 \%$ en adolescentes s/obesidad $(p<.001)$. La hiperinsulinemia se presentó en el 50\% del primer grupo mientras que en el segundo, en $4 \%(p<.001)$. La obesidad incrementó el riesgo de hiperinsulinemia con una razón de momios de (RM) de 23 (IC $95 \%$ : 8,3-68,9) y de por lo menos una dislipidemia $(R M=5,0 ;$ IC95 \%: 2,7-9,2). El nivel de insulina se correlacionó significativamente con IMC $(r=0,57)$, triglicéridos $(r=0,57), \operatorname{VLDL}(r=0,57), \operatorname{HDL}(-0,37)$, relación cintura cadera $(r=0,29)$, colesterol $(r=0,22)$, y LDL $(r=0,13)$.

Conclusiones Se evidenció a la obesidad en adolescentes como factor predisponente para el desarrollo de trastornos metabólicos y la asociación lineal de medidas antropométricas y lípidos con insulina

Palabras Clave: Obesidad, salud adolescentes, riesgo, dislipidemia, insulina, (fuente: DeCS, BIREME).

\section{ABSTRACT}

Objective Determining the prevalence and estimating the risk of obesity for dyslipidemia and hyperinsulinemia in adolescents. The existence of a linear association between 
anthropometric measures, lipids and insulin was also evaluated.

Material and Methods A comparative study was carried out amongst obese (body mass index=BMI >95th percentile for age and gender; $n=120$ ) and non-obese adolescents (BMl <85th percentile for age and sex; $n=120$ ) aged 10-19. A structured questionnaire was used for collecting anthropometric and demographic data. Glucose, insulin and lipid profiles were obtained for each adolescent.

Results Prevalence of at least one dyslipidemia was 56,6 \% among obese adolescents and $20,8 \%$ amongst non-obese ones $(p<.001)$. The former registered $50 \%$ prevalence of hyperinsulinemia, the latter $4 \%(p<.001)$. Obesity increased hyperinsulinemia risk having a 23 odds ratio $(8.3-68.995 \% \mathrm{Cl})$ and for at least one dyslipidemia (OR=5,0; 2,7$9,295 \% \mathrm{Cl})$. Insulin level significantly correlated with $\mathrm{BMI}(\mathrm{r}=0,57)$, triglycerides $(r=0,57)$, $\operatorname{VLDL}(r=0,57)$, HDL $(-0,37)$, waist-hip circumference index $(r=0,29)$, cholesterol $(r=0,22)$, and LDL $(r=0,13)$.

Conclusions Obesity can be considered to be a risk factor for developing metabolic disorders in adolescents. In fact, there was a linear relationship between anthropometric measurement, lipids and insulin. Prevention should focus on improving predisposing environments for obesity amongst families having children and teenagers. Emphasising life-styles and healthy behaviour is essential, as well as training and treatment options for complete care of individuals in this age-group.

Key Words: Obesity, adolescence, risk, dyslipidemia, insulin (source: MeSH, NLM).

$\mathrm{T}$ radicionalmente la obesidad no se consideraba una enfermedad, sino más bien una condición predisponente para el desarrollo de otros padecimientos, por ello no se le registraba como diagnóstico. Sin embargo, la tendencia actual es reconocerla como una enfermedad crónica que constituye un importante problema de salud pública en escala mundial (1). El metabolismo de los lípidos se modifica en el paciente con obesidad siendo la hipertrigliceridemia el trastorno de los lípidos más común. En cambio, el aumento en los niveles de colesterol y lipoproteínas de baja densidad se observa solo en individuos con antecedentes de una historia familiar de hipercolesterolemia (24). Además, la resistencia a la insulina es una característica de la obesidad que condiciona a trastornos metabólicos como hiperinsulinemia y diabetes mellitus y por consecuencia se asocia a un mayor riesgo de morbimortalidad por enfermedades cardiovasculares isquémicas $(5,6)$.

Por otro lado, la adolescencia es una etapa que implica todo un proceso biopsicosocial que influye en diversos aspectos y entre ellos los estilos de vida en donde el adolescente puede tener hábitos alimentarios inadecuados, sedentarismo y consumo de sustancias tóxicas como tabaco, alcohol y otras drogas $(7,8)$. La obesidad en la niñez y adolescencia ha aumentado en forma significativa en los últimos treinta años, tanto en países desarrollados como en 
países en vías de desarrollo. La Encuesta Nacional de Salud y Nutrición de los Estados Unidos (9) (NHANES 2003-2004), representativa de toda la población de ese país reportó en el grupo de adolescentes de 12 a 17 años de edad, que el 17,1 \% tenían sobrepeso, mostrando un importante incremento en los últimos años. En el año 2000, la Encuesta Nacional de Salud en México (ENSA 2000) (10), mostró una prevalencia de sobrepeso en adolescentes de 10 a 19 años de $16,8 \%$ en hombres y $22,7 \%$ en mujeres y la prevalencia de obesidad, de $9,1 \%$ en hombres y 10,6 \% en mujeres. Siendo más frecuente el sobrepeso y la obesidad en las mujeres. Su importancia radica no solamente en su elevada prevalencia sino que desde edades tempranas y de la adolescencia, se pueden encontrar con mayor frecuencia hiperinsulinemia y dislipidemia. Así, se reporta para Turquía en un estudio hecho en niños y adolescentes de 7 a 18 años, insulina elevada en el $43,7 \%$ y dislipidemia en un $55 \%$ (11). También en una muestra de 2000 estudiantes iraníes de 11-18 años, se encontró una prevalencia de 23,7 \% de dislipidemias(12). En México, específicamente en el estado de Jalisco se llevó a cabo un estudio para identificar trastornos metabólicos en adolescentes de 14 a 19 años de edad, y se encontró que el 5,4 \% de la población presentó colesterol total (CT) mayor o igual a $200 \mathrm{mg} / \mathrm{dL}$; el 9,4 \%, lipoproteínas de baja densidad (LDL) mayor a $130 \mathrm{mg} / \mathrm{dL}$; y 17,4 \%, lipoproteínas de alta densidad (HDL) menor a $35 \mathrm{mg} / \mathrm{dL}$. Las mujeres tuvieron concentraciones de CT, LDL y HDL mayores que los hombres (13). Las dislipidemias y el proceso ateroescleroso se inician desde edades tempranas de la vida y constituye un factor de riesgo de gran importancia para la etapa adulta (2).

La resistencia a la insulina es una de las características metabólicas de la obesidad y este fenómeno se encontró en un estudio en niños de en Estados Unidos de América por la American Heart Association y la Universidad de Minnesota $(14,15)$. Otro trabajo realizado en 98 niños con obesidad exógena en Colombia, estableció asociación entre la obesidad y el incremento en los niveles de insulina, colesterol y triglicéridos y una correlación positiva entre los niveles de insulina y el índice de masa corporal (IMC), CT, LDL, triglicéridos y la edad (16). En Jalisco, México; en 352 adolescentes mexicanos encontraron que los niveles de insulina se incrementan con el IMC (17). En el Bogalusa Heart Study(18) donde participaron más de 3500 niños desde 1973, se observaron lesiones ateroescleróticas en etapas tempranas de la vida corroborados en la autopsia de 150 participantes y se encontró una fuerte asociación con los factores de riesgo cardiovascular ante mortem presentando una relación positiva con los niveles de CT, LDL, lipoproteínas de muy baja densidad (VLDL), presión arterial e IMC. En cambio, Odeleye y colaboradores (19) encontraron en una cohorte de 328 niños indios Pima, entre 5 y 9 años una correlación positiva entre la 
concentración de los niveles de insulina y el porcentaje del peso ganado por año.

Bajo el contexto expuesto, el antecedente de la obesidad como riesgo potencial y dado que además de la herencia, hay factores ambientales como hábitos positivos de salud y nivel de desarrollo de una comunidad contribuyentes para desencadenar alteraciones metabólicas relacionadas con la obesidad. Se diseñó este trabajo en uno de los estados más representativos de la región norte del país, en el cual se compara la prevalencia de dislipidemias e hiperinsulinemia entre adolescentes obesos y no obesos y se cuantifica el riesgo de la obesidad para presentar dichos trastornos metabólicos. Asimismo, se evalúa la asociación lineal de medidas antropométricas y trastornos metabólicos específicos con hiperinsulinemia en este grupo de edad.

\section{MATERIAL Y MÉTODOS}

Se realizó un estudio transversal comparativo de adolescentes obesos y no obesos entre 10 y 19 años adscritos a cinco unidades de salud del primer nivel de atención, todas ellas correspondientes a una zona médica de una institución de seguridad social en la ciudad de Monterrey Nuevo León, México. Las zonas médicas son áreas geográficas delimitadas y constituidas por un hospital de segundo nivel de atención y un conjunto de unidades de medicina familiar, cuya red de servicios debe responder a las necesidades de salud de su población. El grupo de estudio con obesidad estuvo integrado por adolescentes con IMC mayor al percentil 95 para la edad y sexo y, el grupo de comparación por adolescentes con IMC menor al percentil 85, también para la edad y sexo. Lo anterior, con base a las Tablas del Centro Nacional de Estadísticas en Salud o National Center for Health Statistics (20), las cuales constituyen el estándar de referencia para el estudio de obesidad. No se consideraron a aquellos adolescentes con alguna enfermedad metabólica como diabetes y a quienes manifestaron consumo excesivo de alcohol en el último mes (21). Además, a adolescentes con embarazo o con ingesta de anticonceptivos orales al momento del estudio. Se eliminaron aquéllos que no acudieron a realizarse las pruebas de laboratorio $(n=80)$ y a quienes les notificaron de manera incompleta el resultado de las pruebas de laboratorio $(\mathrm{n}=4)$.

Los adolescentes se fueron incluyendo en forma consecutiva según requisitos de selección del estudio, al grupo de adolescentes con obesidad y sin obesidad hasta completar el tamaño mínimo de muestra. Se estimaron dos tamaños de muestra considerando los resultados de un estudio piloto local (22). frecuencia esperada de dislipidemia en adolescentes no obesos de $11 \%$ y 
en adolescentes obesos, $46 \%$. Así también para frecuencia esperada de hiperinsulinemia en no obesos de $10 \%$ y en obesos, $33 \%$; resultando una $\mathrm{n}=30$ y 57 para cada grupo, respectivamente; ambas estimaciones con un nivel de confianza de $95 \%$ y una potencia de $80 \%$ (23). Se anticipó una pérdida de $30 \%$, es decir 17 adolescentes más de cada grupo de acuerdo a la $\mathrm{n}=57$. En total, se contó con datos completos de 120 adolescentes obesos y 120, no obesos. A todos los adolescentes se les aplicó un cuestionario estructurado mediante una entrevista. La participación fue voluntaria y previa firma de consentimiento informado del adolescente o de sus padres, en caso de ser menores de edad. Cuando el adolescente no era capaz de responder a alguna de las preguntas, se complementaba con información de alguno de los padres. El instrumento contenía datos generales como edad, sexo y grado máximo de estudios; tiempo en horas por semana dedicado a actividad física, en la escuela y fuera de ella; y tiempo en horas al día dedicadas a ver televisor y práctica de videojuegos. En lo correspondiente a la familia, se investigaron antecedentes de obesidad, diabetes mellitus tipo 2, enfermedad cardiovascular, hipertensión arterial y dislipidemias en padres, hermanos y abuelos. Al término de la encuesta se obtuvo la antropometría de cintura y cadera para evaluar la distribución de grasa; se determinó obesidad central o androide ante una relación de cintura cadera mayor a 0,9 en hombres y 0,8 , en mujeres (24). Por otro lado, se consideró presión arterial óptima y sin riesgo cardiovascular ante una presión arterial sistólica (PAS) igual o menor de $120 \mathrm{~mm} \mathrm{Hg} \mathrm{y} \mathrm{diastólica}$ (PAD), igual o menor de $80 \mathrm{~mm} \mathrm{Hg}$ (25).

Se solicitó al personal de cada una de las unidades de salud participantes que enviaran a los adolescentes con las características mencionadas al módulo establecido específicamente para los fines del estudio. Para estandarizar criterios, técnicas y procedimientos se capacitó al equipo asignado en la toma de medidas antropométricas y tensión arterial con el fin de minimizar errores. Se utilizó una báscula con estadímetro marca BAME de un balance máximo de $140 \mathrm{Kg}$, cinta métrica graduada en milímetros y baumanómetro mercurial portátil tipo integral m1-300 marca ADEX. Una vez concluida la evaluación, se les solicitó a los adolescentes acudir al laboratorio de un hospital de especialidades para la toma de muestra de sangre con el fin de medir insulina, glucosa y perfil de lípidos séricos; se pidió ayuno previo de 13 horas y cena del día anterior libre de grasas. Se usó radioinmunoensayo en la determinación de los niveles de insulina con contador gama, modelo GENESYS Laboratory Technologies, Inc., mientras que para la determinación de lípidos y glucosa, métodos enzimáticos de rutina con aparato analizador automatizado $1 \mathrm{Lab}$ 900, de Instrumentation Laboratory. Se entendió como trastorno metabólico 
ante una o más de las siguientes alteraciones: triglicéridos, $\geq 130 \mathrm{mg} / \mathrm{dL}$; LDL, $\geq 130 \mathrm{mg} / \mathrm{dL}$; VLDL, $\geq 35 \mathrm{mg} / \mathrm{dL}$; HDL, $\leq 35 \mathrm{mg} / \mathrm{dL}$; colesterol $\geq 200 \mathrm{mg} / \mathrm{dL}$; o insulina, $\geq 16 \mu \mathrm{I} / \mathrm{mL}(3,4,26)$. Los adolescentes que resultaron con una 0 más alteraciones metabólicas recibieron instrucciones precisas y envío al médico familiar y al resto de los integrantes del equipo de salud en la unidad médica de adscripción, para el tratamiento correspondiente.

El plan de análisis consistió de estadística descriptiva, la cual incluyó estimación de prevalencias, promedios y desviaciones estándar. Se aplicaron pruebas de $t$ de Student y de z para analizar diferencias entre adolescentes con y sin obesidad, según correspondiera (promedios o porcentajes, respectivamente). Asimismo, se estimaron razones de momios (RM) e intervalos de confianza (IC) de 95\%, y se determinó el coeficiente de correlación de Pearson para evaluar asociación lineal de medidas antropométricas y trastornos metabólicos específicos con hiperinsulinemia.

\section{RESULTADOS}

La edad promedio de los 240 adolescentes fue de $15 \pm 2$,2 años, el 61,7 \% era del sexo femenino, el $81 \%$ estudiaba y el $49 \%$ se encontraba cursando el nivel medio superior. Los grupos de adolescentes con y sin obesidad fueron semejantes con respecto a la distribución de sexo y escolaridad. El promedio de IMC en el primer grupo fue de 32,1 $\pm 4,4$ y en el segundo, de $21 \pm 2,2$ ( $\mathrm{p}<0.001)$; el $58 \%$ de los adolescentes obesos registró una relación de cintura cadera tipo androide en comparación al $19 \%$, de aquellos no obesos ( $\leq \leq 0.0001)$. El análisis por sexo mostró una mayor frecuencia de obesidad central en mujeres que en hombres (65\% vs. $48 \%$, $\mathrm{p}<0.04)$.

En lo que respecta a presión arterial, la media de PAS y PAD fue superior en el grupo de adolescentes obesos (Tabla 1). La prevalencia de cifras tensionales sistólicas no óptimas en el total de adolescentes fue de $25 \%$, y de ellos el $80 \%$ correspondió a adolescentes con obesidad. En lo referente a la PAD, en el $11 \%$ se registraron niveles no óptimos, 92,3\% presentaba obesidad. Por otra parte, se determinó el promedio del total de horas dedicadas a actividad física dentro y fuera de la escuela y se encontró que los adolescentes con obesidad dedicaban $3 \pm 3,5$ horas a la semana, comparado con los no obesos que lo realizaban $5,4 \pm 6,2$ horas por semana $(\mathrm{p} \leq 0.01)$. También el promedio de horas frente a un televisor y práctica de videojuegos fue mayor en el grupo de adolescentes con obesidad ( $3,2 \pm 1,7$ vs. $2,5 \pm 1,5$ horas por día, $\mathrm{p} \leq 0.01$ ). 
Tabla 1. Estadística descriptiva de 240 adolescentes en una institución de seguridad social según condición de obesidad Monterrey, Nuevo León. México

\begin{tabular}{|c|c|c|c|}
\hline \multirow[b]{2}{*}{ Parámetro } & \multicolumn{2}{|c|}{ Obesidad } & \multirow[b]{2}{*}{$\mathrm{p}$} \\
\hline & $\begin{array}{c}\text { Sí } \\
(\mathrm{n}=120)\end{array}$ & $\begin{array}{c}\text { No } \\
(n=120)\end{array}$ & \\
\hline Edad (años cumplidos) & $14,7 \pm 2,3$ & $15,1 \pm 2$ & $\mathrm{NS}^{1}$ \\
\hline Peso (Kilogramos) & $83,7 \pm 16,7$ & $55,3 \pm 9,3$ & $<.001$ \\
\hline Talla (centímetros) & $1,60 \pm .10$ & $1,60 \pm 9,2$ & $\mathrm{NS}^{1}$ \\
\hline Presión arterial sistólica (mm Hg) & $124 \pm 12,6$ & $112,2 \pm 11,5$ & $<.001$ \\
\hline Presión arterial diastólica (mm Hg) & $78,2 \pm 7,5$ & $71 \pm 8,4$ & $<.001$ \\
\hline Glucosa (mg/dL) & $83 \pm 8$ & $82,7 \pm 8,9$ & $\mathrm{NS}^{1}$ \\
\hline Triglicéridos (mg/dL) & $130,5 \pm 67,4$ & $83,8 \pm 39,6$ & $<.001$ \\
\hline LDL-colesterol (mg/dL) & $89,8 \pm 21,5$ & $81,3 \pm 23,6$ & $? .001$ \\
\hline VLDL- colesterol (mg/dL) & $26,1 \pm 13,5$ & $16,7 \pm 7,9$ & $<.001$ \\
\hline HDL- colesterol (mg/dL) & $37,5 \pm 7,4$ & $47,2 \pm 10$ & $<.001$ \\
\hline Colesterol total (mg/dL) & $153,3 \pm 27,7$ & $145, \pm 26,3$ & $? .01$ \\
\hline Insulina $(\mu \mathrm{UI} / \mathrm{mL})$ & $18 \pm 10$ & $7 \pm 3,9$ & $<.001$ \\
\hline
\end{tabular}

Prevalencia de trastornos metabólicos

El único parámetro similar entre adolescentes obesos y no obesos fue la glucosa ( $>>0.05)$. Las cifras promedio de insulina, triglicéridos, colesterol, LDL y LDL fueron estadísticamente superiores ante la presencia de obesidad, mientras que aquellas de HDL resultaron inferiores (Tabla 1). En términos de prevalencia, el $38,7 \%$ de toda la población presentó por lo menos una dislipidemia, en el grupo con obesidad, 56,7 \% y en aquel sin obesidad, 20,8 \% ( $<<0.001)$. La prevalencia de hiperinsulinemia también fue superior en el primer grupo (50 $\%$ vs. $4 \%$, p $<0.001)$. La condición de obesidad incrementó la probabilidad de hiperinsulinemia (RM 23; IC 8,2-68,9) y de por lo menos una dislipidemia (RM 5; 2,71-9,16). La RM para hipertrigliceridemia fue de 4,7 (IC 2,3- 10); hiperlipoproteinemia VLDL, 6.8 (IC 2,1- 24,3); e hiperlipoproteinemia HDL, 5,2 (IC 2,4-11,2). La obesidad no aumentó el riesgo para hipercolesterolemia o hiperlipoproteinemia LDL. La Figura1 muestra en detalle la distribución de frecuencias de los trastornos metabólicos según condición de obesidad. El nivel de insulina se vio afectado en forma directamente proporcional por factores tales como índice de masa corporal, triglicéridos, VLDL, relación de cintura cadera, colesterol y LDL. En cambio, las cifras de HDL le produjeron un efecto inversamente proporcional. En la Tabla 2 se muestran los coeficientes de correlación correspondientes.

El análisis de antecedentes familiares en adolescentes con algún tipo de dislipidemia ( $\mathrm{n}=93$ ) mostró que el $22 \%$ de los mismos contaba con por lo menos 
un familiar directo con trastorno metabólico en los lípidos; 70 \%, con diabetes mellitus tipo 2; $44 \%$, con hipertensión arterial; y $61 \%$, con obesidad. En lo referente a los adolescentes con hiperinsulinemia $(n=65)$, el $71 \%$ presentó antecedentes familiares de diabetes; 69 \%, de obesidad; 51 \%, de hipertensión; y 21,5 \%, trastornos en los lípidos.

Tabla 2. Correlación de antropometría y lípidos con insulina en 240 adolescentes de una institución de seguridad social. Monterrey, Nuevo León. México

\begin{tabular}{lcc}
\hline \multicolumn{1}{c}{ Factor } & $\begin{array}{c}\text { Coeficiente de } \\
\text { correlación de } \\
\text { Pearson }\end{array}$ & Valor de $\mathrm{p}$ \\
\hline Índice de masa corporal & 0,57 & $<.01$ \\
Relación cintura cadera & 0,29 & $<.01$ \\
Triglicéridos & 0,56 & $<.01$ \\
VLDL & 0,56 & $<.01$ \\
HDL & $-0,37$ & $<.01$ \\
LDL & 0,13 & $<.05$ \\
Colesterol total & 0,22 & $<.01$ \\
\hline
\end{tabular}

Prevalencia de trastornos metabólicos según sexo

En la Tabla 3 se encuentran resultados detallados estratificados por sexo. La prevalencia de adolescentes con cifras sistólicas $>120 \mathrm{~mm}$ Hg fue más alta ante obesidad independientemente de ser mujer u hombre. En cambio, la frecuencia de cifras diastólicas no óptimas fue mayor sólo en mujeres obesas. Finalmente, el efecto de obesidad persistió para hiperinsulinemia, hipertrigliceridemia, hiperlipoproteinemia VLDL y HDL, tanto en el sexo masculino como femenino. Así también, permaneció la ausencia de asociación para colesterol y LDL.

\section{DISCUSIÓN}

La obesidad en adolescentes es un factor predisponente para el desarrollo de trastornos metabólicos como se evidenció en el presente trabajo de investigación, en el cual la mayoría contaba con 16 años de edad, estaba inscrito en la escuela media superior y era del sexo femenino. La prevalencia y las posibilidades de riesgo para hiperinsulinemia y dislipidemia fueron superiores y estadísticamente significativas en adolescentes con obesidad en comparación a los no obesos y predominó en los primeros, la obesidad central. Precisamente del acumulo de grasa central se derivan las principales complicaciones de la obesidad como aquellos riesgos cardiovasculares y psicosociales mencionados en otros estudios (27). 


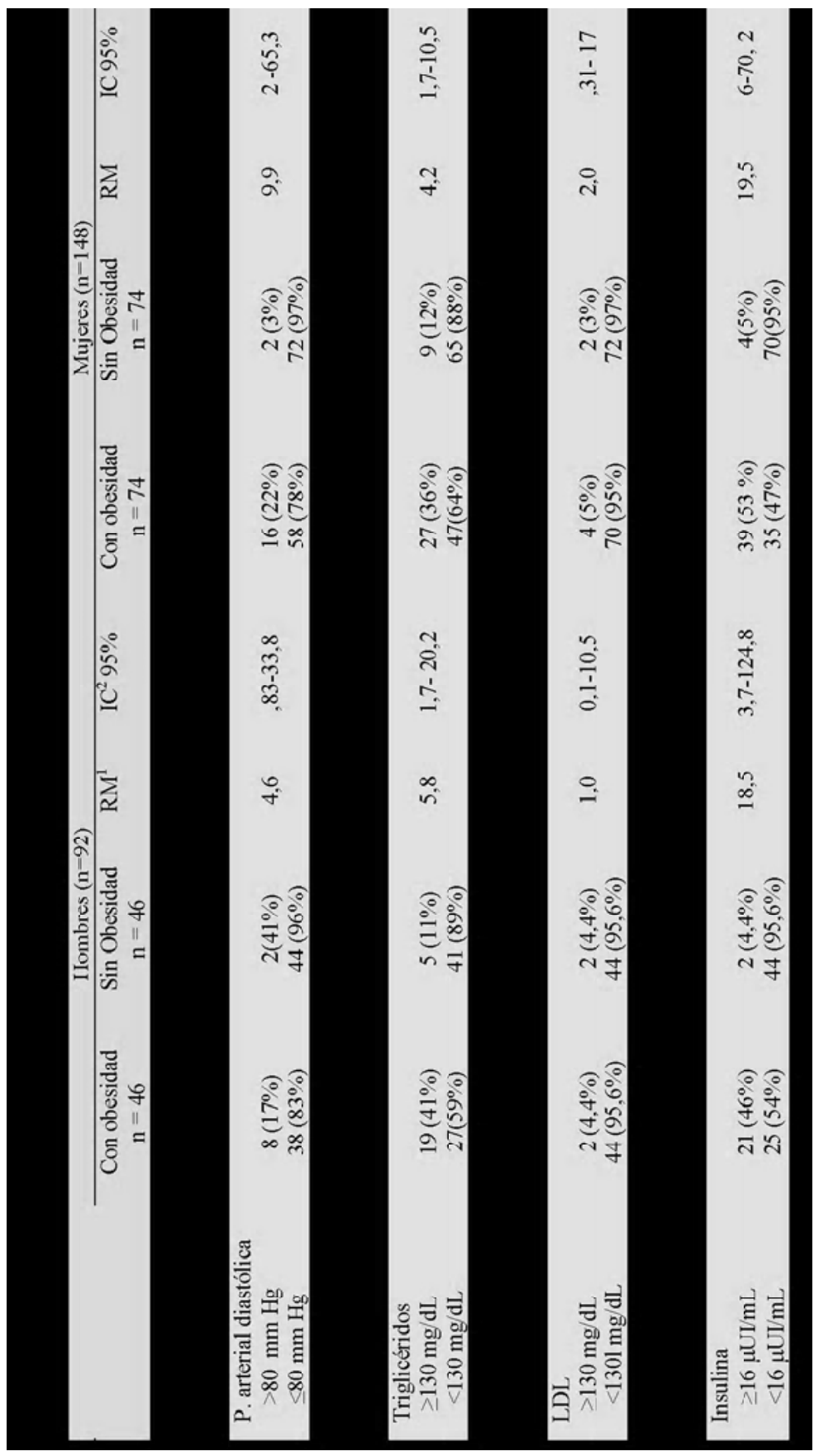


Las dislipidemias son factores de riesgo modificables para prevenir la ateroesclerosis que pueden ser fácilmente diagnosticadas en la niñez y adolescencia. En este estudio, poco más de uno de cada dos adolescentes obesos presentó por lo menos una dislipidemia, dicha prevalencia es similar a lo reportada para niños turcos obesos en edad de la pubertad (11). Por otro lado, no se encontraron diferencias entre hombres y mujeres, a pesar de que este último se ha considerado de mayor riesgo en otros estudios $(28,29)$. De las cinco dislipidemias estudiadas, la hipertrigliceridemia fue la más frecuente tal y como era de esperarse, ya que la misma se encuentra directamente vinculada con la obesidad, además tiene concordancia con lo ya descrito por otros autores (2-4). Fue notoria la baja prevalencia de hipercolesterolemia e hiperlipoproteinemia de baja densidad y la similitud en frecuencia para obesos y no obesos. Una posible explicación sería el hecho de que estas alteraciones del metabolismo del colesterol y LDL generalmente se encuentran en individuos con antecedentes de historia familiar, además de la obesidad por sí misma (2-4). Por otro parte, solo 20 de c/100 presentó antecedente familiar directo con dislipidemias aunque se anticipaba una mayor frecuencia en adolescentes con estos trastornos. Sin embargo, más de la mitad de los mismos reconoció tener algún miembro de la familia con obesidad o diabetes. Lo anterior se debe tomar con cautela ya que los datos dependieron del conocimiento del adolescente o de sus padres, sobre la existencia de dichos padecimientos en la familia, por lo que la prevalencia correspondiente pudiera estar subestimada.

El papel que desempeña el aumento de los niveles de insulina en el desarrollo de los factores de riesgo cardiovascular ha generado recientemente mucho interés. La hiperinsulinemia en niños y adolescentes ha sido asociada con disminución de HDL, y elevación de triglicéridos y de presión arterial sistólica. Plourde (30) en adolescentes caucásicos de Canadá observó correlaciones positivas de IMC con insulina, triglicéridos, colesterol, HDL y LDL tanto en hombres como en mujeres. Asimismo, Awadalla y colaboradores (16), en Colombia encontraron una correlación positiva entre niveles de insulina e IMC, colesterol, LDL y triglicéridos en niños y adolescentes entre 8 y 13 años de edad. En el presente trabajo también se mostró una correlación positiva entre obesidad (IMC y concentración de grasa central) e insulina, así como trastornos de lípidos aunque para lipoproteínas cardioprotectoras HDL, la correlación fue negativa. Es importante considerar que la asociación causal entre obesidad e insulina no está clara todavía. Es posible pensar que la hiperinsulinemia misma es la que predispone a la obesidad, incluso Odeleye y colaboradores 19 documentan en su trabajo realizado con niños indios Pima que esta alteración puede ser examinada como predictora de obesidad. Se requiere continuar con esta línea de investigación 
utilizando diseños de estudio que permitan esclarecer la direccionalidad de estas asociaciones.

En cuanto al efecto de la obesidad en presión arterial, se observaron prevalencias superiores de niveles no óptimos PAS de y PAD en aquellos individuos obesos. Esto puede ser atribuido a la hiperinsulinemia sostenida, la cual asociada a la obesidad afecta al sistema nervioso simpático y daña la función endotelial (31). En lo concerniente a la actividad física, se esperaría que por la edad inherente a la adolescencia se practicara algún deporte. Fueron notoriamente escasas las horas que en promedio se ocupaban a la semana en actividad física por el adolescente con obesidad. También en estudiantes iraníes en adolescencia, el 66,6 \% no realizaba algún tipo de actividad física (12). La importancia de este hallazgo radica en las futuras complicaciones como se muestran en este estudio y en aquel realizado por Ramírez y colaboradores (17) en una población de adolescentes mexicanos de 14 a 19 años, en donde concluyen que la inactividad física se relaciona estrechamente con la obesidad, obesidad central y altos niveles de insulina.

Los resultados aquí obtenidos pueden ser extrapolables con las debidas precauciones. La frecuencia de obesidad central y de trastornos en los lípidos como está ligada a factores genéticos y a patrones de consumo de alimentos llega a variar según el origen étnico de la población bajo estudio. Sin embargo, el efecto de la obesidad sobre la insulina y lípidos ha observado hallazgos equivalentes en poblaciones tan distintas como colombiana (16), turca (11), caucásica-canadiense (30), además de la mexicana. La consistencia de resultados en distintos grupos étnicos con diversidad en estilos de vida permitirá el avance de conocimiento en esta área con beneficios incuestionables para la sociedad. Se pueden anticipar soluciones de marcada dificultad, especialmente porque se conjunta la fuerza de la voluntad para el cambio de hábitos con la falta de conciencia de riesgo en la población adolescente, incluso por parte del personal de salud. Con frecuencia no se ve a la obesidad como una enfermedad per se; sin embargo, su diagnóstico y tratamiento y mejor aún su prevención, son la clave para evitar enfermedades costosas en salud y en dinero. La diabetes, hipertensión y otras enfermedades cardiovasculares secundarias a concentraciones elevadas de lípidos, ocupan no solamente los primeros lugares como motivo de consulta sino también de causas de muerte, incluso de pérdidas de vida prematuras de jóvenes en países desarrollados y subdesarrollados. En ese sentido, los servicios de salud deben mantenerse vigentes y proactivos para dar respuesta a las necesidades a este grupo de riesgo que es la población adolescente* 
Agradecimientos. Los autores agradecen al personal directivo y médico de la Unidad Médica de Altas Especialidades (UMAE) No. 34 y de las Unidades de Medicina Familiar (UMF) No. 26,28,35,37 y 39 por el apoyo brindado durante el desarrollo del proyecto.

\section{REFERENCIAS}

1. World Health Organization. Obesity: Preventing and Managing the Global Epidemic. WHO Obesity Technical Report Series 894. Geneva, Switzerland: World Health Organization; 2000.

2. Kanani PM, Sperling MA. Hyperlipidemia in Adolescents. Adolesc Med 2002; 37:37-52.

3. Gullesserian T, Widhalm K. Effect of rapeseed oil substituting diet on serum lipids and lipoproteins in children and adolescents with familial hypercholesterolemia. Journal of the American College of Nutrition 2002; 21:103-108.

4. Expert Panel on Blood Cholesterol Levels in Children and Adolescents. National Cholesterol Education Program. Report of the Expert Panel on Blood Cholesterol Levels in Children and Adolescents. Paediatrics Supplement. 1992; 89:525-584.

5. Janssen I, Katzmarzyk P, Srinivasan S, Chen W, Malina R, Bouchard C , et al. Utility of childhood BMI in the prediction of adulthood disease: comparison of national and international references. Obesity Research 2005; 13:11061115.

6. Viner RM, Segal TY, Lichtarowicz-Krynska E, Hindmarsh P. Prevalence of the insulin resistance syndrome in obesity. Arch. Dis. Child. 2005;90:10-14.

7. Casanueva E, Kaufer-Horwitz M, Pérez-Lizaur AB, Arroyo P. Nutriología Médica. 2a ed. México:Editorial Médica Panamericana; 2001.

8. Raftopoulos C, Bermingham M, Steinbeck K. Coronary heart disease risk factors in male adolescents, with particular reference to smoking and blood lipids. Journal of Adolescent Health. 1999; 25:68-74.

9. Ogden CL, Carroll MD, Curtin LR, Mc Dowell MA, Tabak CJ, Flegal KM. Prevalence of overweight and obesity in the United States, 1999-2004. JAMA. 2006; 295:1549-1555.

10. Celis de la Rosa A . La salud de adolescentes en cifras. Salud Pública Méx. 2003; 45 (1):153- 166.

11. Atabeck ME, Pirgon O, Kurtoglu S. Prevalence of metabolic syndrome in obese Turkish children and adolescents. Diabetes Res Clin Pract.2006;72 (3):315321. 
12. Kelishadi R, Sadri G, Tavasoli A, Kahbazi M, Roohafza H, Sadeghi M. et al. Cumulative prevalence of risk factors for atherosclerotic cardiovascular diseases in Iranian adolescents: IHHPO-HHPC. Jornal de Pediatría. 2005; 81: 447-453.

13. Grupo de Estudio de Insulinemia en Adolescentes. Concentración de insulina y lípidos séricos en adolescentes de preparatoria en Guadalajara, México. Salud Pública Méx. 2003; 45103-107.

14. Steinberger J, Daniels S. Obesity, insulin resistance, diabetes and cardiovascular risk in children. Circulation. 2003; 107:1448-1453.

15. Steinberger J. Diagnosis of the metabolic syndrome in children. Curr Opin Lipidol. 2003; 14:555-559.

16. Awadalla S, Castillo JM. Relación entre los niveles de insulina basal, el perfil lipídico y el índice de masa corporal en los niños con obesidad exógena. Actual Pediatr FSFB 2000; 10:126-129.

17. Ramírez-López G, González-Villalpando C, Sánchez-Corona J, Salmerón-Castro J, González-Ortiz M, Celis-de la Rosa A, Valles Sánchez V. Weight, Physical Activity, and Smoking as Determinants of Insulinemia in Adolescents. Archives of Medical Research. 2001; 32:208-213.

18. Berenson G, Srinivasan S, Bao W, Newman W, Tracy R, Wattigney W. Association between multiple cardiovascular risk factors and atherosclerosis in children and young adults. For the Bogalusa Heart Study. N Eng J Med. 1998; 338:1650-1656.

19. Odeleye OE, de Courten M, Pettit DJ, Ravussin E. Fasting hyperinsulinemia is a predictor of increased body weight gain and obesity in Pima Indian children. Diabetes 1997; 46:1341-1345.

20. National Center for Health Statistics [Internet]. Disponible en http://www.cdc. gov/nchs/about/major/nhanes/growthcharts/charts.htm . Consultado 2 de abril de 2007.

21. Morales-García JIC, Fernández-Gárate IH, Tudón-Garcés H, Zárate-Aguilar A. Prevalencia de consumo riesgoso y dañino de alcohol en derechohabientes del Instituto Mexicano del Seguro Social. Salud Pública Méx. 2002; 44:113121.

22. Marcos NJ, Hinojosa JO, Tamez HE, Treviño JH. Obesidad en la adolescencia. Su asociación con hiperinsulinemia y dislipidemias. Archivos de Investigación Pediátrica en México.1999; 1:3-6.

23. Dean AG, Arner TG, Sangay S, Sunki GG, Friedman R, Lantinga M, Zubieta JC, Sullivan KM, Smith DC. Epi Info 2000, a database and statistics program for public health professionals for use on Windows 2000. Centers for disease Control and Prevention, Atlanta, Georgia, USA; 2000. 
24. Styne DM. The Paediatric Clinics of North America. Childhood and adolescent. Obesity. 2001; 48:823-847.

25. Secretaría de Salud Norma Oficial Mexicana. NOM-030-SSA2-1999. Para la prevención, tratamiento y control de la hipertensión arterial. México: Secretaría de Salud; 1999.

26. Wallace TM, Matthews DR. The assessment of insulin resistance in man. Diabetic Medicine. 2002; 19: 527-534.

27. Sweeting H, Wrigth Ch, Minnis H. Psychosocial correlates of adolescent obesity, "slimming down" and "becoming obese". Journal of Adolescent Health 37; 2005: 409-417.

28. Burrows R, Bargueño M, Leiva L, Ceballos X, Guiller I, Gattas V, et al. Perfil metabólico de riesgo cardiovascular en niños y adolescentes obesos con menor sensibilidad insulínica. Rev Med Chile 2005; 133:795-804.

29. Hoffman RP, Vicin P, Sivitz WI, Cobellio C. Pubertal adolescent male-female differences in insulin sensitivity and glucose effectiveness determined by the one minimal model. Paediatric Research 2000; 48:384-388.

30. Plourde G. Impact of obesity on glucose and lipid profiles in adolescents at different age groups in relation to adulthood. BMC Family Practice 2002; 3:18-31

31. Fisch SR, Tamborlane TB, Banyas B, Allen K. Prevalence of impaired glucose tolerance among children adolescent with market obesity. N Engl. J. Med. 2002; 346:802-810. 